\title{
The Influence of Stubborn Agents in a Multi-Agent Network for Inter-Team Cooperation/Negotiation
}

\author{
Eugene S. Kitamura \\ Department of Computer Science, \\ National Defense Academy \\ Yokosuka, Japan
}

\author{
Akira Namatame \\ Department of Computer Science, \\ National Defense Academy \\ Yokosuka, Japan
}

\begin{abstract}
When teams interact for cooperation or negotiation, there are unique dynamics that occur depending on the conditions. In this paper, a multi-agent system is used under the restrain of a network structure to model two teams of agents interacting for a common consensus, however with the presence of stubborn agents. The networks used were a minimum dumbbell network and two scale-free networks joined together. The network topology, which is a global characteristic, along with the presence of conflicting stubborn agents, can cause various conditions that affect teamwork in cooperation or negotiation. Notable characteristics revealed are boundary role persons (BRPs), lack of unity, need for a third party moderator, coalition formation, and loyalty of the BRP dependent on the distance from the core ideology of the team. Both local and global characteristics of network structures contribute to such phenomenon. The modeling method and corresponding simulation results provide valuable insight for predicting possible social dynamics and outcome when planning
\end{abstract} cooperation/negotiation tactics.

Keywords-Multi-agent system; consensus problem; stubborn agents; complex network; dumbbell network; Laplacian matrix; boundary role person; coalition formation

\section{INTRODUCTION}

\section{A. Team dynamics and consensus}

The success of an organization depends on how effective its team of agents operate, whether in business or in other social settings. Thus, it is natural for organizational managers to have an interest in understanding group dynamics. A team, as opposed to an individual, is a conglomeration of diverse talents and perspectives, and allows distribution of tasks for efficient operations [1, 2]. Formation of a robust team with diverse agents is especially valued today in business to cope with rapidly changing technological and global markets [3, 4]. However, because of its collective nature of talents and personalities, a team of agents may be exposed to internal conflict and noise in addition to external ones during cooperation or negotiation with another team of agents. Such perturbations can result in unintended consensus dynamics within the team. How does the formation of consensus opinion depend on the locations of stubborn agents in the social network? We would like to be able to predict the outcomes such that we can control or avoid certain situations during the formation of consensus.

To have a holistic view of team dynamics, a manager must consider the micro and macro properties of a team. Micro properties considered important are planning, which is subdividing tasks among agents, coordination, which is the synchronization of agent actions and its continuous monitoring for assessment, and communication, which is information exchange among agents [5]. These qualities are micro properties since they are defined for and executed by the agents that form a team. On the other hand, macro properties are the network structure that embeds the agent interactions or patterns of relationships within a team [6,7]. With such network topological aspect, the team dynamics is not only determined by agent characteristics or local interaction rules, but also determined by a global structural restraint. This network formed by the agent team can be considered a macro property since this property ignores the characteristics of each agent and it affects the entire community. For managers and team members to understand and predict team consensus dynamics, they need to observe and evaluate both their micro and macro properties. Failure of such considerations may result in disagreements and formations of coalitions that may impede the team's initial goal.

Consensus dynamics in general have been studied in computer science and control theory for a long time [8]. In a network of agents, consensus is formed when all of the agents agree to a certain parameter state. A consensus algorithm performs a consensus procedure where agents exchange their parameter status among its local neighbors in the agent network so that they may eventually reach an agreement. The analytical foundation of consensus problems for networked systems was presented by Olfati-Saber and colleagues [9]. The consensus problem is also related to synchronization. Synchronization phenomena are seen in diverse settings such as neurons firing, laser cascades, biological cycles, opinion formation, and in chaotic dissipative systems in general. Many such phenomena in nature are realized by synchronization in a constructive manner. However, forming synchronization in a harmful context can cause detrimental effects such as collapse of bridges or causing epileptic seizures $[10,11]$. In order to manage such destructive outcomes by synchronization, Louzada [12] studied the use of contrarians to suppress undesired synchronization. Contrarians systematically dephase from the oscillation of their nearest neighbors. Louzada compared the use of contrarians with access to local and global information and concluded that contrarians with local interactions are enough for the most efficient influence. Additionally, when the interacting neighbor number (degree distribution) is relatively even and contrarians are placed at 
highly connected nodes, the synchronization dephasing performance is significantly improved.

In this paper, instead of contrarians that desynchronize with its neighbors, the presence of stubborn agents that refuse to form consensus with the rest of the agents in the network is considered. Consensus formation of agents under the restriction of a network is observed. In particular, the network used is structured so that it can represent two teams interacting. Two stubborn agents with opposing opinions are used to represent a disagreement in the network. The terms agent and node are often used interchangeably, where an agent may have a stronger social/multi-agent system aspect whereas a node may have a complex network connotation.

\section{B. Consensus formation with stubborn agents}

Studies of consensus and synchronization under a network structure is important for understanding its dynamics as seen in the previous section. However, there is no guarantee that all of its agents in the network will cooperate. Some agents may lead the team to form a consensus by influencing the rest of the agents or some agents may mislead the consensus formation to an unintended final state. These situations may occur in diverse scenes such as socio-economic situations [13], rendezvous strategies [14], average consensus [15], and sensor deployment [16]. Gupta [17] studied possible scenarios of agent "failure" in the context of distributed algorithms used in the above research presented. The first failure condition is a stopping failure [18] where an agent blacks out and stops communicating with the other agents. The second failure condition is when an agent value becomes stuck at a fixed state. Fagnani [19] showed that if the rest of the agents are non-stubborn, then the agents would converge to this fixed value agent. The third failure condition is when an agent continuously changes its state to erroneous values at every time step, either intentionally or unintentionally $[18,20]$.

In a social context of stubborn agents, Acemoglu [21] studied the spread of misinformation by using "forceful" agents in an agent value averaging model. Forceful agents are not completely stubborn, but under particular conditions they have a strong influence on some of their neighbors such that the terminal consensus value is diverted from the original consensus value without the forceful agents. Spread of misinformation is quantified by measuring the magnitude of this divergence. Instead of forceful agents, Yildiz [22] presented a consensus behavior study with two stubborn agents with opposing opinions with different fixed agent values, using a classical voter model $[23,24]$. In this model, the stubborn agent does not affect all of its neighbors, but rather chooses one neighbor randomly, and instead of taking an average value between the two agents, the neighbor agents adopt their neighbor's value. They found that with the presence of opposing stubborn agents, the opinions among the agent society disagree and fluctuate. Finally, Acemoglu [25] uses an inhomogeneous stochastic gossip model of communication. The agents update their belief as a convex combination of their own belief and the belief of their neighbor at the same time step. In addition to finding that consensus process fluctuates and never converges, they demonstrated that in a general network topology the intermediate agents between the stubborn agents take terminal values which are linear interpolations of the two stubborn agents' beliefs.

In the three above mentioned investigations, they consider dumbbell (barbell) graphs to observe the influence of either single, double, and triple stubborn agents on the rest of the non-stubborn agents. Their dumbbell graphs have two or three cliques of arbitrary agent number. Two small interacting teams can be modeled with a dumbbell graph with complete graphs connected by bridging agents and a link. The smallest complete graphs with three agents are a sufficient condition for a negotiation team [26], since working in small teams allow more flexibility, agility, and adaptability [27, 28]. Modeling of interactions between larger organizations may not be so simple due to its hierarchical structure or the involvements of many sub-divisions in the organization. However, considering that even large organizations would have a small scale working unit or a representative team for negotiations [29], a dumbbell teamwork interaction model is a reasonable and practical consideration. Due to the linking property of a dumbbell graph, special roles are played by bridging agents and link. In graph theoretical terms, these bridging agents have a higher betweenness centrality [30]. In a team cooperation or negotiation context, these bridging agents are called boundary role persons (BRPs) [31] and have a unique role in the team [32]. Later, a modeling of larger teams is also considered by using two scale-free networks connected.

With the presence of one or two stubborn agents, the following questions are investigated: what is the overall dynamics of the society of agents with the presence of stubborn agents? How opinion dynamics are affected with the presence of two stubborn agents holding completely opposite opinions? These are investigated under the constraint of a network structure. First, the time progression of the consensus formation process or stabilization process (if the dynamics is non-convergent) for a minimum dumbbell graph is demonstrated. The time progression shows the various patterns of approach resulting in diverse final outcomes depending on the location of the stubborn agents. Second, the notion of small team dynamics is extended to a team with a larger population and a greater distance of the BRP from the core ideology (stubborn agent) of its team. Two identical scale-free networks with a larger population are used instead of minimum complete graphs. The final states settle as a linear interpolation of agent locations as observed in [25]. However, the time progression shows that the consensus is formed among the local tree modules first, then a global steady state is reached. The presence of two stubborn agents results in the formation of coalitions. The model also supports that the farther away physically and psychologically the BRP is from the core team ideology (stubborn agent), the BRP agent opinion becomes closer to its opponent group [33].

\section{ANALYTICAL FOUNDATION}

\section{A. Consensus protocol}

The average consensus problem makes use of spectral graph theory and matrix theory $[15,34,35]$. Consider a symmetric (undirected) connected graph $G=(V, E)$ which is the network topology that restricts the agent interactions, where 
$V$ is a set of nodes and $E$ is a set of edges. The linear continuous-time consensus protocol used for the network agent dynamics is $[15,35]$,

$$
\dot{x}_{i}=\sum_{j \in N_{i}} \alpha_{i j}\left(x_{j}(t)-x_{i}(t)\right)
$$

where $x_{i}$ and $x_{j}$ is the state value of agent $i$, and its network neighbor $j$ respectively, $\alpha_{i j}$ is the weight of agent $i$ on agent $j$, $N_{i}$ is the set of neighbors connected to agent $i$, and $t$ is time or iterations. Here, $\alpha_{i j}=\alpha_{j i}$ for all $i, j$, since the graph $G$ is undirected. Consensus is achieved when the agent values converges to a common value based on (1), i.e.,

$$
x_{1}=x_{2}=\cdots=x_{n}=\alpha,
$$

where $\mathrm{n}$ is the total number of agents in the network. When there are no stubborn agents or other interferences in the agent network, the value taken by (2) can be expressed by the following equation,

$$
\alpha=\frac{1}{n} \sum_{i=1}^{n} x_{i}(0)
$$

where $x_{i}(0)$ is the initial agent values at $t=0$. In other words, without any bias the consensus converges asymptotically to the average of the initial values. The Laplacian matrix $L$ of the graph network $G$ is explained below. The Laplacian matrix is defined as the following,

$$
\mathbf{L}=\mathbf{D}-\mathbf{A},
$$

where $\mathbf{D}=\operatorname{diag}\left(d_{1}, d_{2}, \cdots, d_{n}\right)$ is the diagonal matrix with elements $d_{i}=\sum_{j} a_{i j}$ and $\mathbf{A}$ is the binary adjacency matrix ( $n \times n$ matrix) with elements $a_{i j}$ for all $i, j$ where $a_{i j}$ is 1 if agent $i$ and agent $j$ is connected or 0 if they are disconnected. Then the dynamics of the system in (1) can be expressed as

$$
\dot{x}=-\mathbf{L} x(t) \text {. }
$$

\section{B. Stubborn agents}

The presence of stubborn agents affects the formation of consensus, and the deviation of this consensus opinion dynamics depends on the network structure and the location of the stubborn agents on that structure. Fagnani [19] shows the analytical framework of consensus dynamics over networks including regular agents and stubborn agents. Consider a symmetric connected graph $G=(V, E)$ introduced earlier. Separate $V=S \cup R$ with the understanding that agents in $S$ are stubborn agents not changing their state while agents in $R$ are regular agents whose state modifies with time according to the consensus dynamics. With the presence of stubborn agents, the whole consensus dynamics can be described by the relation

$$
x(t+1)=P x(t) .
$$

When the elements in $V$ are ordered in such a way that elements in $R$ come first, the matrix $P$ will exhibit the block structure:

$$
P=\left[\begin{array}{cc}
Q_{1} & Q_{2} \\
0 & I
\end{array}\right]
$$

By splitting the state vector accordingly, $x(t)=\left(x_{R}(t), x_{S}(t)\right)$, the dynamics shown below is obtained.

$$
\begin{aligned}
& x_{R}(t+1)=Q_{1} x_{R}(t)+Q_{2} x_{S}(t) \\
& x_{S}(t+1)=x_{S}(t)
\end{aligned}
$$

Notice that $Q_{1}$ is a sub-stochastic matrix, i.e., all row sums are less than or equal to 1 . There is at least one row whose sum is strictly less than one, which is the row corresponding to a regular agent connected to a stubborn one. Using the connectivity of the graph, this easily implies that there exists $t$ such that $\left(Q_{1}\right)^{t}$ has the property that all its rows have sum strictly less than one. This immediately yields that the matrix is asymptotically stable. Therefore,

$$
x_{R}(t) \rightarrow x_{R}(\infty) \text { for } t \rightarrow+\infty
$$

with the limit opinions satisfying the relation which is equivalent to

$$
\begin{aligned}
& x_{R}(\infty)=Q_{1} x_{R}(\infty)+Q_{2} x_{S}(0) \\
& x_{R}(\infty)=\left(I-Q_{1}\right)^{-1} Q_{2} x_{S}(0) .
\end{aligned}
$$

Regular agents settle asymptotically to opinions that are the convex combinations of the opinions of stubborn agents. The above analysis shows, that if all stubborn agents are in the same state $x$, for instance there is just one stubborn agent, then, consensus is reached by all agents in the opinion of the stubborn agent $x$. However, typically, consensus is not reached. Few examples modeled by simulation are discussed below. The initial state value of each agent is set as,

$$
x_{i}(0)=i(i=1,2, \cdots, N)
$$

When each agent updates her state value according to (1), the state of each agent converges to a constant value, which is the average of the initial values of all agents. The consensus dynamics in (1) converge to the average of the initial states of all agents without stubborn agents. Additionally, the consensus dynamics profile is different depending on the location of the two stubborn agents.

C. Networks

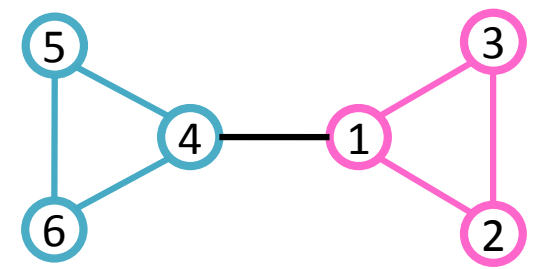

Fig. 1. A diagram of a dumbbell graph. Each clique is composed of 3 agents. The graph contains total of 6 nodes and 7 links. Since each clique consists of a minimum complete graph with 3 nodes, this network is considered a minimum dumbbell network 
In the first experiment, we use a dumbbell network. A dumbbell network is a structure with two cliques connected by a single link (Fig. 1). "Simplest" here means the network consists of minimum number of nodes. The definition of a negotiating team is a group of two or more interdependent persons who collaborate to achieve a mutual goal through negotiation and they all attend the bargaining table [36]. The cliques used here consist of three nodes all connected to each other to form a complete graph. One arbitrarily node is chosen from each clique and these nodes are connected by a bridging link. These bridging nodes play a unique role in that they have an absolute influence on their own clique and the greatest influence to the opposite clique when stubborn agents are located at these bridging nodes.

In the second experiment, two scale-free networks were connected. This network was generated to model a greater number of nodes and greater distance between the stubborn agent and the bridge node. Two identical scale-free networks with 50 nodes were generated and the nodes with the least closeness centrality or the most "remote" nodes (Fig. 4, node 50 and 100) were connected. The node chosen for each stubborn agent is the highest alpha-centrality [37] for each scale-free network (nodes 1 and 51). The overall network forms a line network with modular tree structures attached to the nodes on the main line network. A line network is the simplest structure to model the "psychological distance" between the organizational core and the boundary. The entire network is symmetric since two identical networks were connected at identical locations.

\section{RESULTS}

First, the results from the simplest case of a dumbbell network are presented. On the dumbbell network, where agents are grouped with their clique network, local consensus is promoted in each clique network at first. After that, the global consensus is formed via the bridge link as seen in Fig. 2. Progressively increasing and evenly spaced initial values are assigned to the agents from agent 1through 6 as seen in Fig. 2, except for stubborn agents. Stubborn agents are assigned a node value of either 0 or 100 , two extremely opposite values. Without stubborn agents, the agent converges asymptotically, eventually reaching a consensus value of the average of agent values as indicated in (3). Smaller values are assigned to agents in the violet color clique and larger values are assigned to agents in the blue color clique in Fig. 1.

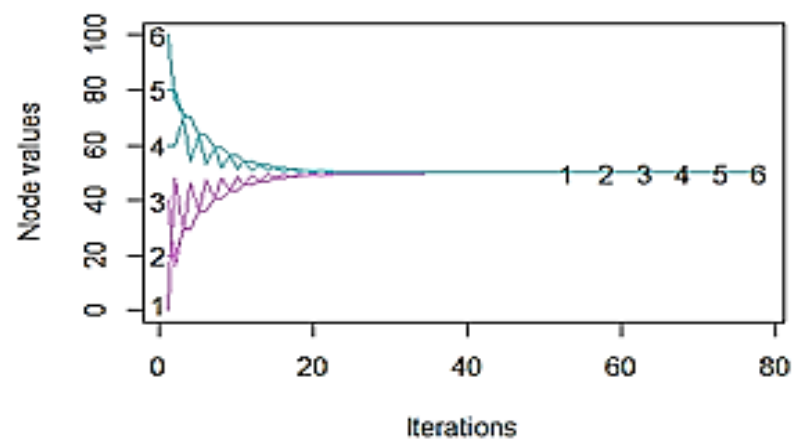

Fig. 2. A consensus progression diagram for the dumbbell graph in Fig. 1. The initial values assigned to agents in each clique are 0 to 50 to one clique and the 51 to 100 to the other clique
With the presence of stubborn agents, three types of consensus behavior were noticed for a dumbbell network topology with single and double stubborn agents. The first type is for a single stubborn agent located at node 1 in Fig. 1 (case 1, Fig. 3(a)). This is a trivial solution where all of the agent opinions converge to the stubborn agent, regardless of the location of the stubborn agent. Such dynamics has been analytically modeled by Fagnani [19]. The second type is for two stubborn agents in the same clique. There are two possibilities for this type. The first possibility is when the stubborn agents are located at nodes 2 and 3 so none of the stubborn agents are the bridge agent (case 2, Fig. 3(b)). The rest of the regular agents from both cliques converge to an intermediate value. For the second possibility, the stubborn agents are located at nodes 1 and 2, one of the stubborn agents being a bridge agent (case 3, Fig. 3(c)). The regular agent (agent 3) in the same clique will converge to an intermediate value between the two stubborn agents and all of the agents in the opposite clique without stubborn agents will converge to the bridging stubborn agent node 1 . In this case, the regular agents trapped between the stubborn agents converge to an average value between the two stubborn agent values.

The third type is again for two stubborn agents, but one located in each clique. In general, the regular agents in a clique will approach the stubborn agent in their respective cliques, however, under the influence of the stubborn agent of the opposite clique. When one of the stubborn agents is located at node 1 bridge agent (case 4, Fig. 3(d)), this stubborn agent shuts out the rest of the agents in its clique from any influence from the opposite clique. One could say that the stubborn agent at the bridge node "dominates" its clique. Similarly, this bridging stubborn agent will influence the agents in the opposite clique, where its influence will be the strongest with the bridging regular agent node 4 , then with indirect and reduced influence with agents beyond the bridge agent of the opposite clique (agent 6), except for the stubborn agent (agent $5)$. In this case, the regular agents with indirect influence converge to a value between its stubborn agent (agent 5) and the bridging agent (agent 4 ) as in case 3 .

When the two stubborn agents are located at the bridge nodes of their respective cliques, agents 1 and 4 (case 5, Fig. $3(\mathrm{e})$ ), the regular agents will converge to the stubborn agent of their own clique. The regular agents in one clique will never reach a consensus with the regular agents in the opposite clique and vice versa because all influence from the opposite clique is blocked by the stubborn agent at the bridge node of its own clique.

If none of the bridge agents are stubborn (case 6, Fig. 3(f)), the regular agents are not topologically restricted to the influence of a particular stubborn agent. The bridge agents receive the largest influence of the opposite clique and the rest of the regular agents converge to a value between their bridge agent and the stubborn agent in their clique. The regular agents do not converge with the stubborn agent in their clique because of the influence of the stubborn agent in the opposite clique. In general, when the number of agents in the cliques increases while maintaining a complete graph structure, the values of regular agents within the clique tend to attract or "bond" closer together. 


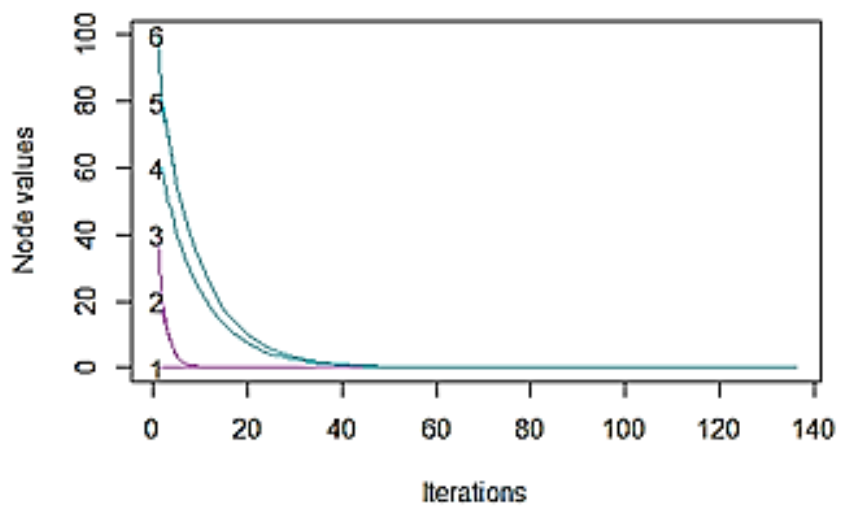

(a)

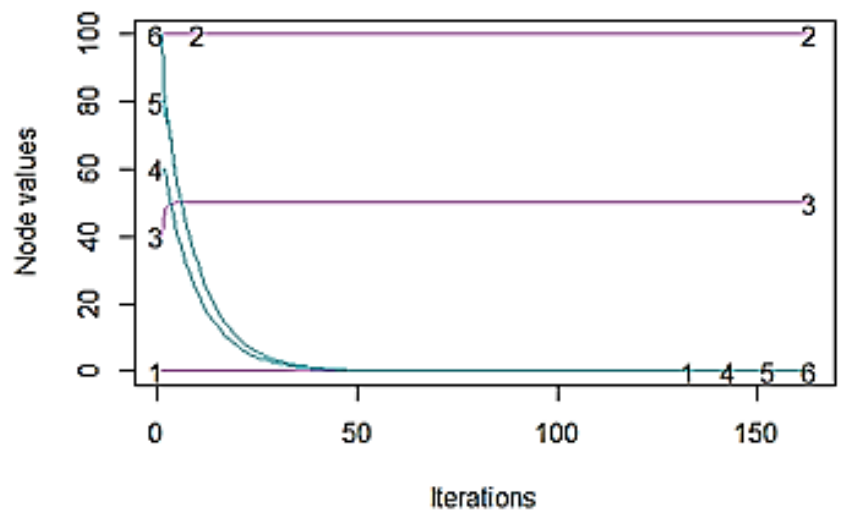

(c)

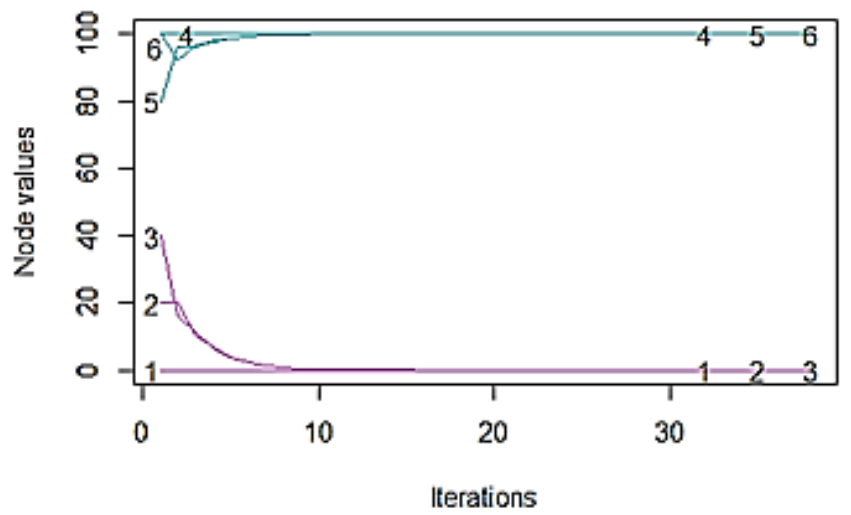

(e)

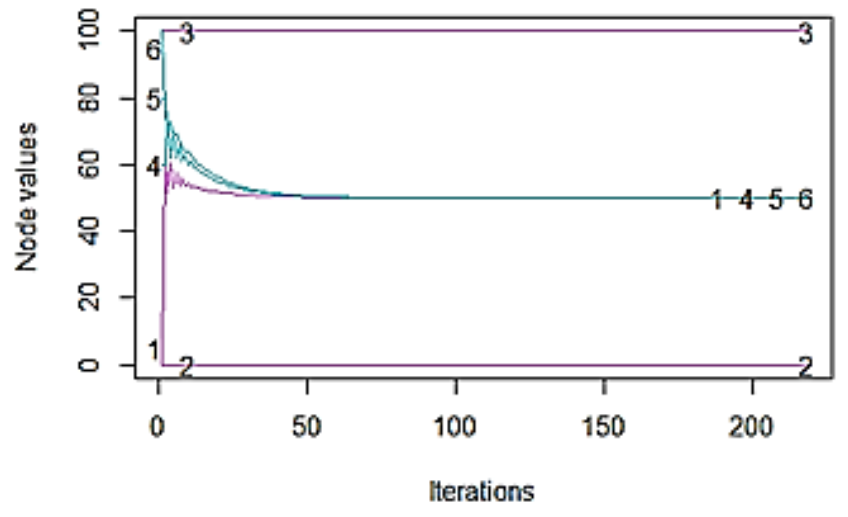

(b)

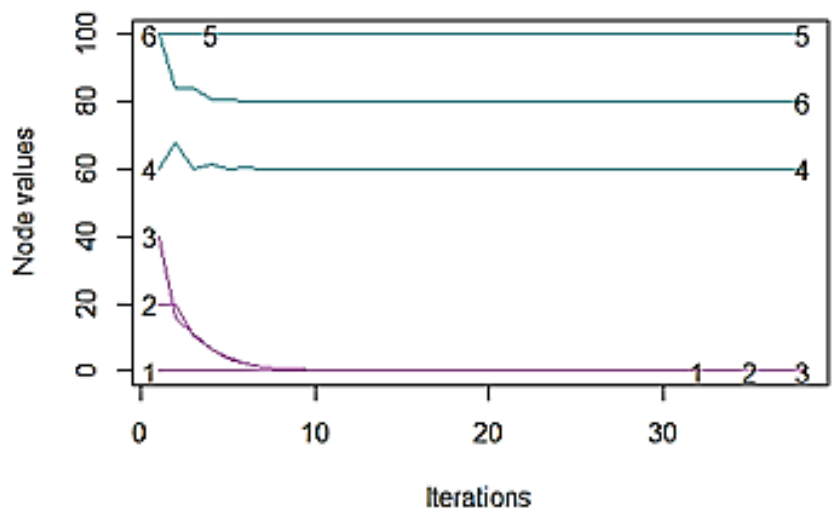

(d)

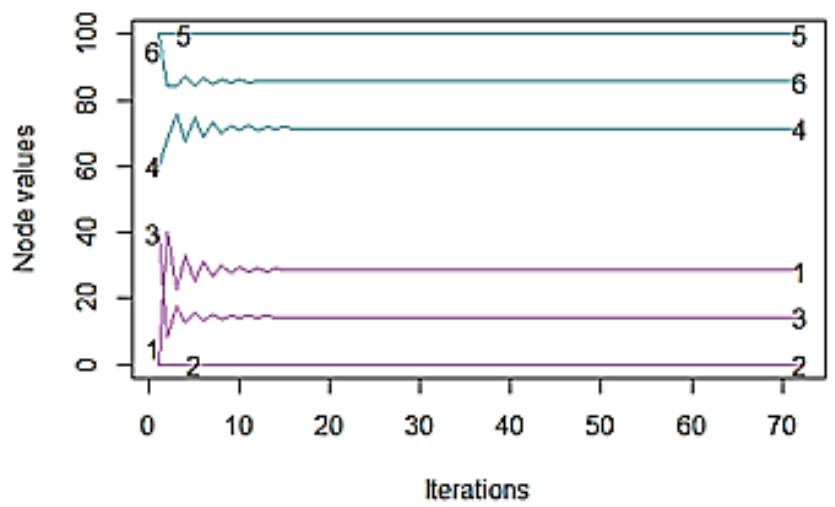

(f)

Fig. 3. Graph with the change in the agent value. $x$-axis is time step and $y$-axis is node value. Node number for stubborn agents are (a) node 1, (b) nodes 2 and 3 , (c) nodes 1 and 2, (d) nodes 1 and 5, (e) nodes 1 and 4, and (f) nodes 2 and 5. The node numbers are written on the graph lines

Not only that, the value of the regular agents, except for the bridging agents, converge to a single value. The bridging agents of the opposite cliques attract each other so they do not converge completely with the rest of the regular agents in their respective clique. This is also seen in case 4 (Fig. 3(d)). The following is the result from the scale-free network structure (Fig. 4). The number of time steps it took for the agent values to stabilize ( $\Delta x_{i}=10^{-9}$, which is the difference in the values between two time steps) is close to 6,300 time steps (Fig. 5). The dynamics with two stubborn agents show that consensus is formed locally among the tree module structures branching from the "main" link path connecting the two stubborn agents (a line network). The branching agent is a hub agent for its community. Consensus is formed among such local modules first. When there are no stubborn agents, a global convergence will follow. 


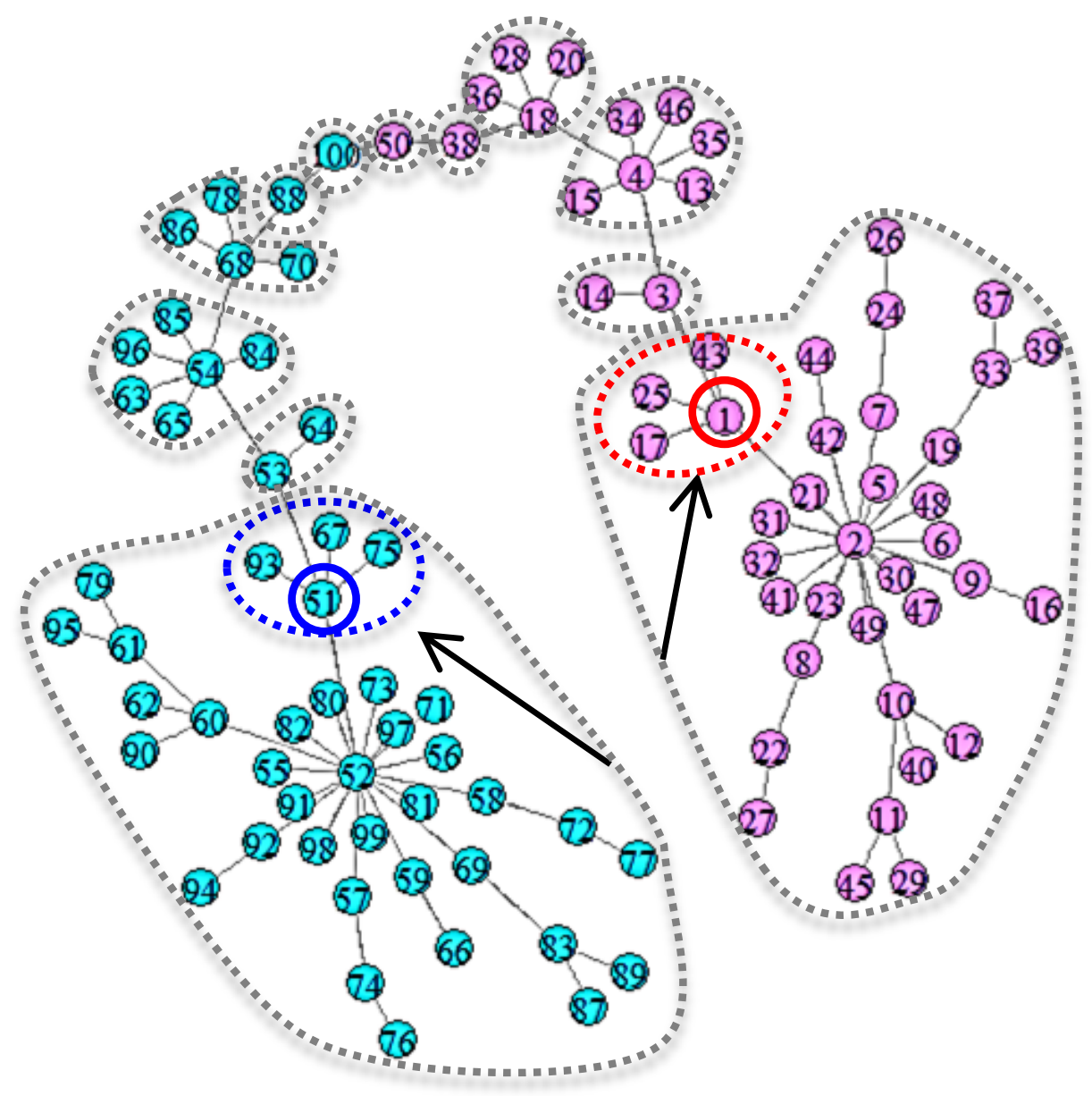

Fig. 4. Two scale-free networks connected by the most "remote" nodes. The agents forming local consensus are grouped by the gray dotted line. The stubborn agents are marked by the red and blue circles. The red and blue dotted lines are the local consensus initially formed by the tree module community with the stubborn agent as the hub. The black arrow shows that the larger consensus community eventually forms a consensus with the stubborn agent community, a dynamics which can also be observed in Fig. 5

However, when there are two stubborn agents located at the opposite ends of the line network, local consensus never merges together. In fact, the values taken by the local module consensus are essentially the hub agent number evenly dividing the agent value range (Fig. 5), a linear interpolation indicated by [25]. Consequently, the number of consensus settling values equals the number of hub agents along the main link path between the two stubborn agents, in addition to the two stubborn agent values. The order of consensus values associated with the hub communities is consistent with its order along the main link path that connects the two stubborn agents.

One consensus dynamics to notice is the convergence of two tree modules (Fig. 4). Initially, the tree modules of agents 1 and 2 form a consensus among their own modules. Then as time progresses, the consensus of these two modules converges. This occurs because agent 1 is a stubborn agent and all agents in the agent 2 module are regular agents. Therefore all agents in the agent 2 module form a consensus first. Then as time passes, they form a consensus with the stubborn agent 1 . The result in the other half of the scale-free network follows a symmetric dynamics with agents 51 and 52 .

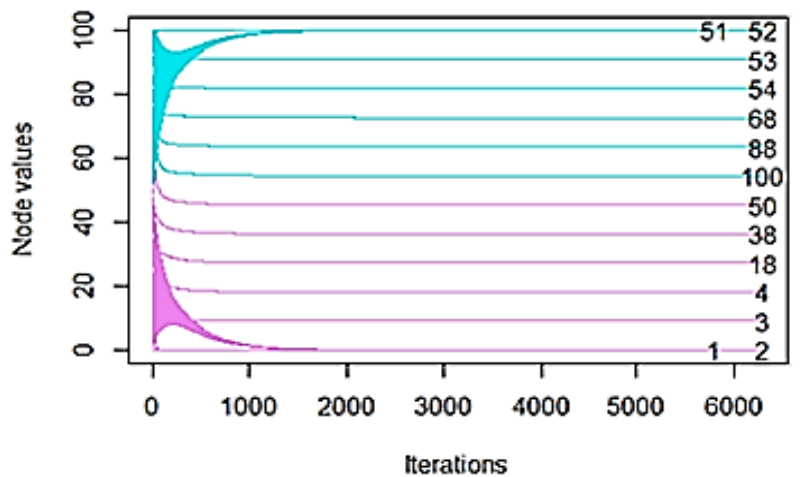

Fig. 5. Graph with the change in the agent value. $x$-axis is time step and $y$ axis is node value. The node numbers written on the agent values are the stubborn agents $(1,51)$ and the hub agents forming a consensus among their tree module along the main link path between the two stubborn agents

When two stubborn agents are located at the neighboring most "remote" linking agents at nodes 50 and 100, the two scale-free networks form a consensus only within their network community, i.e., the regular agents are attracted to the stubborn agent in their own community. 
This is analogous to case 5 in the dumbbell topology dynamics. The value of consensus of each team depends on the value of their respective stubborn agent. Due to the limited node degree of these stubborn agents (agents 50 and 100), the consensus takes almost 10 times longer (about 59,000 time steps) than when the stubborn agents are placed at the nodes with the highest alpha-centrality (agents 1 and 51).

\section{DISCUSSION}

This paper investigates the behavior of agents under the influence of opposite opinions or conflicting signals. The agents are caught between dynamics that aggregate and dynamics that are biased. When working in a team, the members must cope with both internal and external noise. Additionally, such local dynamics are met with the global constraint of a network. In the context of team negotiations in business or social settings, an analyst needs to take into account the impact of the social and organizational environments within which agent teamwork dynamics are embedded [38]. The agents' roles, the team characteristics, and agent relationship networks are critical sources of influence within a negotiations setting in particular [39], but this can be applied to other teamwork dynamics as well.

\section{A. Minimum dumbbell team structure}

The dumbbell network settings can be used to model a negotiation or a collaborative situation between two teams of agents on a single project. In case 1 , a leader with a clear objective, vision, and resource can guide the two teams and the rest will follow (Fig. 3(a)). In case 2, two of the members of the same team may have opposite opinions (two stubborn agents), which is an intra-group conflict situation [40] (Fig. 3(b)). In a real world setting, the teammates are 'not on the same page,' 'confused about goals,' and possess 'conflicting interest within the team' [26]. This may happen when the agents represent different departments and have different goals to achieve. Since the members in the teams must maintain their respective teams (maintain the clique structure), the rest of the agents in both teams take an intermediate opinion and collaborate or find a common ground (form a local consensus) (Fig. 3(b), agents 1, 4, 5, 6). The solution to such intra-team conflict is to have prior preparation for a common agenda [26].

In case 3, the team with stubborn agents will be divided in 3 opinion values, the two stubborn values and the intermediate value of the agent that is connected to the two strong opposite opinions (Fig. 3(c)). If one of the bridge members has a strong vision and leadership (agent 1) and if all the agents in the opposite team are regular agents (agents 4, 5, 6), the opposite team will form an agreement with the bridging member of the team with stubborn agents (agent 1). The bridge agent is known as a boundary role person (BRP). Because such BRPs are often closer to the opposite team, physically and psychologically, they naturally share a relationship with the BRPs of the opposite team. This can potentially weaken the bond of the BRP with their own team [32]. After all, it is often preferable that negotiators have an understanding of and act in a way to accommodate the opponent's needs [31]. The influence of the other team is stronger with individualistic cultures in the occident than in the collectivist cultures in the orient, because BRP of the individualistic cultures are less bound to their own team [26].

In case 4 , one of the bridging members, a BRP, is a stubborn agent or a leader (Fig. 3(d), agent 1). However, there is a strong opposing opinion in the other team which is not a BRP (agent 5). The members of the BRP leader team follow the BRP leader. Although the BRP leader's opinion has an influence on the team members of the other team, where the strongest influence is on the BRP of the other team and less influence on the non-BRP regular agents, the BRP leader cannot completely win over all the agents in the other team due to the opposition member (agent 5) in the other team. This case is a good example to show the extent of influence an agent would have depending on the location in a global network topology. One stubborn agent is a BRP (agent 1) and the other stubborn agent (agent 1 ) is not. In a team negotiation context, it is known that the quality of the negotiation outcome may be in favor of the BRP that is closer in psychological distance to the core ideologies (stubborn agent) of its own team. Perry [33] has concluded that when one of the negotiators is psychologically distant from their own team, the bargaining outcomes should fall in between the outcomes obtained when the negotiators are either close or far away psychologically. This outcome can be seen in the time progression of Fig. 3(d). Additionally, there is a profound significance to this in a corporate setting. As mentioned earlier, a BRP is located in a privileged position in that it is the only agent to reach the opposite team. Therefore, BRP is an agent that has access to internal as well as external (other corporate) information. Assume that BRP initially does not have any hierarchical authority or a legitimate basis of power. It is just another employee. However, during the time of environmental turbulence for business, due to its unique position in the interaction network of having both internal and external information, the BRP has the potential ability to cope with and absorb uncertainty, and channel or control the flow of information to its own company [41]. Since "power" is defined as an inverse of dependency [42], a BRP could gain power which initially would not have had if it were not a BRP. In fact, the BRP could intentionally generate conflict to maintain the company's dependency on itself and thus keep its power. This is seen again later in this paper.

In case 5 , the connecting members of the two teams have opposite opinions (Fig. 3(e), agents 1 and 4). Since the regular agent members (agents 2, 3, 5,6) of their respective teams are flexible and obedient to their strong leader, the two teams would not reach a consensus and therefore could not collaborate. This is a situation where the intensity of conflict is high. If a consensus of two teams must be achieved, the two teams may need a mediator or an arbitrator [43]. Mediation is where a third party makes a recommendation that is mutually acceptable based on information about the two teams. Arbitration is where the two teams commit to following the third party recommendation. The arbitrator has enforcing means. Both mediators and arbitrators are a third party that intervenes in the negotiation process [44]. In case 6, when there are oppositions by non-bridge members in each team (Fig. 3(f), agents 2 and 5), even though the members are attracted by the other team, they would not form a single consensus. Rather, the 
opinion of the members would range between the two extreme opinions, where the intermediate BRPs would receive the greatest influence from the other team. Although in case 5 a consensus was formed in their respective cliques, the formation of coalitions is beginning to appear in case 4 and 6 . This is more so when we have a tree module line network, which is discussed below.

\section{B. Scale-free network team structure}

So far, a model of interaction of small scale teams was considered. The location of stubborn agents and the role of the BRP were examined. When the number of agents increases in a team, there are two things that can change from a smaller group. The first is, as the number of agents increase, there will be more opinions and cultures. The second is, the BRP at the boundary surface may become more distant from the core ideology of their team held by a strong leader (stubborn agent).

First considering the cultural aspect, when the number of cultural diversity increases, the team is exposed to the possibility of forming coalitions within the team. Coalition is a subunit of a team that is consisted of two or more members whose intentions are to fulfill their own goals instead of the goals of the host team [45]. Coalition is said to form among those who have similar social/cultural traits. Here, social/cultural traits are individualism/collectivism, masculinity/femininity, etc. [46]. When the team is culturally homogeneous or completely heterogeneous, the likelihood of coalition formation is less. However, when the diversity of cultures is intermediate, a team is exposed to the possibility of coalition formation [47]. The presence of a coalition in a team can be unproductive because it may cause internal conflict and distancing among members [48]. Coalition makes the team more complex and unstable.

Assuming various cultures exist in a team, such cultures may be modeled as a sub-modular structure in the overall network, as seen in the hub and branch module structure in Fig. 4. When there are two stubborn agents, the agents and their cultural groups positioned between the two stubborn agents may hold varying degrees of sentiments depending on their location. During the consensus procedure, these network modules form a consensus within their cultural module. If there are no stubborn agents or if there is a single stubborn agent, these modules form a global consensus after they have established a local consensus as we have seen in Fig. 2. This two stage convergence is seen also for agents not in between, but "outside" the two stubborn agents, such as agents 2 and 52 community who eventually form a consensus with the agents 1 and 51 community. However, when there are two opposing state values (agent 1 and 51), the local culture modules between the two stubborn agents never converge with the other culture modules due to the influence of the opposing stubborn agents. Thus, multiple coalitions are formed within the team.

Finally, considering the distance of the BRPs from the core ideologies of their team, the farther away they are from the core and closer to the periphery near their opposite team, there is the risk of the negotiation outcome become less attractive for its own team where the BRP belongs and more preferable for the opposite team. There are two reasons why this may happen. First, because of the distance of the BRP from the core, the negotiator BRP may have an ambiguous understanding of the team's goal because of conflicting information. The second reason is the BRP may set incongruent goals of their team, either unintentionally due to lack of information or intentionally in order to create uncertainty in the situation so that they would be relied upon more by their organization and thus gaining more power. The farther away the BRP is from the core, this trend is stronger [33]. The terminal values taken by the BRP agents in Fig. 5 reflect this situation. The closer the BRP is to their opposite team and farther away they are from their respective "core" stubborn agent values, the BRP values of both teams become closer.

\section{CONCLUSION AND SUMMARY}

A multi-agent system was used to model the interaction of agents under the restrain of a team structure. The agents have an averaging protocol for consensus formation. However, not all agents are cooperative. The team interaction structures used were a minimum dumbbell network and two scale-free networks connected. All possible outcomes of the minimum dumbbell structure with two stubborn agents were interpreted in a team cooperation/negotiation setting. As indicated by previous literature, the bridging agents or boundary role persons have a unique role to serve. Its position on the network showed that it has a strong influence over the other agents. When stubborn agents are located at both bridging nodes, the agents are strongly united with their team that it is recommended to involve a third party to moderate or arbitrate.

The scale-free line network with tree modules showed the possibility of coalition formation based on the cultural diversity in the team. Such coalition formation dynamics can be seen in the way modular networks form a consensus, that local consensus is formed first and then a global consensus is reached. This shows the influence of both local and global topology that influences the overall characteristics of the network dynamics. The branching agents from the main line nodes can be considered to have the same culture/attitude/mentality. This network can also model the psychological distance between the boundary role person and the core ideology of the team. The farther away they are from the core, and more cultural values there are in between, their ideology becomes closer to the other team. Such a model can be used not only for commercial settings, but also in a political setting in society, between two extreme policies.

\section{REFERENCES}

[1] T. H. Cox, Cultural Diversity in Organizations: Theory, Research and Practice. San Francisco, CA: Barrett-Koehler, 1993.

[2] S. Jackson, K. E. May, and K. Whitney, "Understanding the dynamics of diversity in decisions making teams," in Team Effectiveness and Decision Making in Organizations, R. A. Guzzo and E. Salas, Eds. San Francisco, CA: Jossey-Bass, 1995, pp. 204-261.

[3] D. R. Illgen, J. R. Hollenbeck, M. Johnson, and D. Jundt, "Teams in organizations: From I-P-O models to IMOI models," Annual Review, vol. 56, pp. 517-543, 2006.

[4] A. W. Richter, M. A. West, R. van Dick, and J. F. Dawson, "Boundary spanners' identification, intergroup contact, and effective intergroup relations," The Academy of Management Journal, vol. 49, pp. 12521269, 2006.

[5] P. L. Curşeu, P. Kenis, J. Raab, and U. Brandes, "Composing effective teams through team-dating," Organ. Stud., vol. 31, pp. 873-894, 2010. 
[6] D. J. Watts and S. H. Strogatz, "Collective dynamics of small-world networks," Nature, vol. 393, pp. 440-442, 1998.

[7] A. L. Barabási, Linked: The New Science of Networks, Cambridge, MA: Perseus Publishing, 2002.

[8] A. Pikovsky, M. Rosenblum, and J. Kurths, Synchronization: A Universal Concept in Nonlinear Sciences, Cambridge, UK: Cambridge University presses, 2003.

[9] R. Olfati-Saber, J. A. Fax, and R. M. Murray, "Consensus and cooperation in networked multi-agent systems", in proc. of IEEE, vol. 95, no. 1, pp.215-233, 2007.

[10] M. Barahona, "Synchronization in small-world systems," Physical Review Letters, vol. 89, 2002.

[11] X. F. Wang and G. Chen, "Synchronization in scale-free dynamical networks: Robustness and fragility", IEEE Transactions on Circuits and Systems I: Fundamental Theory and Applications, pp.54-62, 2001.

[12] V. H. P. Louzada, N. A. Araujo, and H. Herrmann, "How to suppress undesired synchronization," Scientific Reports 2, no. 658, 2012.

[13] D. Acemoglu, G. Como, F. Fagnani, and A. Ozdaglar, "Opinion fluctuations and disagreement in social networks," Mathematics of Operation Research, vol. 38, no. 1, pp. 1-27, 2013.

[14] M. Ji, G. Ferrari-Trecate, M. Egerstedt, and A. Buffa, "Containment control in mobile networks," IEEE Transactions on Automatic Control, vol. 53, no. 8, pp. 1972-1975, 2008.

[15] R. Olfati-Saber and R. M. Murray, "Consensus problems in networks of agents with switching topology and time-delays," IEEE Transactions on Automatic Control, vol. 49, no. 9, pp. 1520-1533, 2004.

[16] J. Cortes, S. Martinez, T. Karatas, and F. Bullo, "Coverage control for mobile sensing networks," IEEE Transactions on Robotics and Automation, vol. 20, no. 2, pp. 243-255, 2004.

[17] V. Gupta, C. Langbort, and R. Murray, "On the robustness of distributed algorithms," in proc. of the 45th IEEE Conference on Decision and Control, 2006.

[18] N. Lynch, Distributed Algorithms. San Mateo, CA: Morgan Kaufmann Publishers, 1996.

[19] F. Fagnani, "Consensus dynamics over networks," 2014.

[20] L. Lamport, R. Shostak and M. Pease, "The Byzantine generals problem,' ACM Transactions on Programming Languages and Systems, vol. 4, no. 3, pp. 382-401, 1982.

[21] D. Acemoglu, A. Ozdaglar, and A. ParandehGheibi, "Spread of (mis)information in social networks," Games and Economic Behavior, vol. 70, no. 2, pp. 194-227, 2010.

[22] E. Yildiz , D. Acemoglu, A. Ozdaglar, A. Saberi, and A. Scaglione, "Discrete opinion dynamics with stubborn agents," 2011.

[23] R. A. Holley and T. M. Liggett, "Ergodic theorems for weakly interacting infinite systems and the voter model," The Annals of Probability vol. 3, no. 4, pp. 643-663, 1975.

[24] P. Clifford and A. Sudbury, "A model for spatial conflict," Biometrika, vol. 60 , no. 3, pp. 581-588, 1973 .

[25] D. Acemoglu, G. Como, F. Fagnani, and A. E. Ozdaglar, "Opinion fluctuations and disagreement in social networks," Math. Oper. Res. vol. 38, no.1, pp. 1-27, 2013.

[26] K. Behfar, R. Friedman, and J. M. Brett, "The team negotiation challenge: defining and managing the internal challenges of negotiating teams," IACM 21st Annual Conference Paper, November, 2008.

[27] D. S. Alberts and R. E. Hayes, Power to the edge: Command...control... in the information age. Washington: CCRP, 2003.

[28] D. S. Alberts, "Agility, Focus, and Convergence: The Future of Command and Control," The International C2 Journal, vol. 1, pp.1-30, 2007.
[29] S. Brodt and L. Thompson, "Negotiating teams: A levels of analysis approach," Group Dynamics-Theory Research and Practice, vol. 5, no. 3, pp. 208-219, 2001.

[30] M. Barthélemy, "Betweenness centrality in large complex networks," The European Physical Journal B - Condensed Matter and Complex Systems, vol. 38, issue 2, pp. 163-168, 2004.

[31] R. E. Walton and R. B. McKersie, A Behavioral Theory of Labor Negotiations: An Analysis of a Social Interaction System. New York: McGraw-Hill, 1965.

[32] J. S. Adams, "The structure and dynamics of behavior in organization boundary roles," in Handbook of Industrial and Organizational Psychology, M. D. Dunnette, Ed. Chicago: Rand McNally, 1976, pp. 1175-1199.

[33] J. L. Perry and H. L. Angle, "The politics of organizational boundary roles in collective bargaining," The Academy of Management Review, vol. 4, no. 4, pp. 487-495, October 1979.

[34] L. Xiao and S. Boyd, "Fast linear iterations for distributed averaging," Systems \& Control Letters, vol. 53, no.1, pp. 65-78, 2004.

[35] W. Ren, R. W. Beard, and E. M. Atkins, "A Survey of Consensus Problems in Multi-agent Coordination," in proc. of the American Control Conference, 2005.

[36] L. Thompson, E. Peterson, and S. Brodt, "Team negotiation: an examination of integrative and distributive bargaining," J. Per. Soc. Psychol., vol. 70, pp. 66-78, 1996.

[37] P. Bonacich and P. Lloyd, "Eigenvector-like measures of centrality for asymmetric relations," Social Networks, vol. 23, pp. 191-201, 2001.

[38] R. M. Kramer and D. M. Messick, Negotiation as a Social Process. Thousand Oaks, CA: Sage, 1995.

[39] M. J. Gelfand and J. M. Brett, The Handbook of Negotiation and Culture. Stanford, CA: Stanford University Press, 2004.

[40] K. Jehn, "A qualitative analysis of conflict types and dimensions in organizational groups," Administrative Science Quarterly, vol. 42, pp. 530-557, 1997.

[41] R. E. Spekman, "Influence and information: An exploratory investigation of the boundary role person's basis of power," Academy of Management Journal, vol. 22, no. 1, 1979.

[42] R. Emerson, "Power dependence relations," American Sociological Review, vol. 22, pp. 31-41, 1962.

[43] M. Goltsman, J. Hörner, G. Pavlov, and F. Squintani, "Mediation, arbitration and negotiation," Journal of Economic Theory, vol. 144, no. 4, pp. 1397-1420, 2009.

[44] R. J. Lewicki, S. E. Weiss, and D. Lewin, "Models of conflict, negotiation and third party intervention: A review and synthesis," Journal of Organizational Behavior, vol. 13, no. 3, pp. 209-252, May 1992.

[45] J. K. Murnighan, "Organizational coalitions: structural contingencies and the formation process," in Research on Negotiation in Organizations, vol. 1, R. J. Lewicki, B. H. Sheppard, and M. Bazerman, Eds. Stanford, CT: JAI Publishing, 1986, pp. 155-173.

[46] G. Hofstede, Culture's Consequences, International Differences in Work-related Values, Beverly Hills, CA: Sage, 1980.

[47] X. Guo and J. Lim, "Negotiation support systems and team negotiations: The coalition formation perspective," Information and Software Technology, vol. 49, pp. 1121-1127, 2007.

[48] S. M. B. Thatcher, K. A. Jehn, and E. Zanutto, "Cracks in diversity research: The effects of diversity faultlines on conflict and performance," Group Decis. Negotiation, vol. 12, pp. 217-241, 2003 